Please cite this article as:

A. Romero, J. González, M.J. Deen, J.A. Jiménez-Tejada, Versatile model for the contact region of organic thin-film transistors, Organic Electronics, (2020), $77,105523$.

(C2020. This manuscript version is made available under the CC-BY-NC-

ND 4.0 license

http://creativecommons.org/licenses/by-nc-nd/4.0/

Digital Object Identifier:

10.1016/j.orgel.2019.105523

Source:

https://www.sciencedirect.com/science/article/pii/S1566119919305506?via\%3Di $\underline{\text { hub }}$ 


\title{
Versatile Model for the Contact Region of Organic Thin-Film Transistors
}

\author{
A. Romero ${ }^{\mathrm{a}, \mathrm{b}}$, J. González ${ }^{\mathrm{a}}$, M.J. Deen ${ }^{\mathrm{c}}$, J. A. Jiménez-Tejada ${ }^{\mathrm{b}, *}$ \\ ${ }^{a}$ Departamento de Arquitectura y Tecnología de Computadores, CITIC-UGR, Universidad de Granada, Granada 18071, Spain \\ ${ }^{b}$ Departamento de Electrónica y Tecnología de Computadores, CITIC-UGR, Universidad de Granada, Granada 18071, Spain \\ ${ }^{c}$ Department of Electrical and Computer Engineering, McMaster University, 1280 Main Street West, Hamilton, Ontario L8S 4K1, Canada
}

\begin{abstract}
Contact effects in organic thin film transistors (OTFTs) remain an important problem to be solved in these devices. Therefore, the correct physio-chemical modeling of the contact regions in OTFTs is necessary. In this work, a standard model for the contact region of OTFTs is proposed. It is a versatile model that describes the current-voltage characteristics of different kinds of contacts. It reproduces the behavior of Schottky barrier or space-charge limited contacts. It is a simple unified model since only a single parameter is necessary in order to distinguish between both kinds of contacts. The model is easily integrated in a generic compact model for the current-voltage characteristics of OTFTs. The resulting compact model, used in combination with an evolutionary parameter extraction procedure, allows to extract the intrinsic parameters and the current-voltage curves at the contact of single short-channel transistors. There is no need to use transistors with multiple channel lengths to accurately characterize the contact region or the active channel of the transistor. The model is tested with published experimental data of OTFTs with Schottky barrier or space-charge limited contacts. Finally, the method has been used as a diagnostic tool to analyze how an ammonia sensor reacts to different concentrations of the ammonia gas. Interestingly, alterations in the contact region have been detected when the gas concentration varies, transforming the space-charge limited contact of a pristine OTFT into a Schottky barrier contact under the exposure of gas.
\end{abstract}

Keywords: organic thin-film transistor, transistor model, contact effects, parameter extraction, multi-objective optimization, evolutionary algorithms, ammonia sensor

\section{Introduction}

Organic thin-film transistors (OTFTs) are among the most popular technologies for lightweight, low-cost, flexible, and stretchable electronics [1-4]. Recently, significant advances were made in improving their important limitations such as a poor mobility [5-8], high process variability [9] or nonflexible electrodes [10]. Nevertheless, even these state-of-theart OTFTs are often limited by their contact regions. In the past, the impact of contact effects was treated by many research groups [11-21]. Recently, many efforts were also done on reducing the contact effects, such as testing different materials as contact electrodes [22, 23], the insertion of layers at the metal-semiconductor interface [24, 25], or the use of molecular doping [26]; and also on working toward an universal compact model of organic transistor technologies that includes the contact effects [27].

In order to examine the effect of the contacts, characterization methods to extract the contact resistance are necessary. These methods must work together with transistor models,

\footnotetext{
This work was supported by projects MAT2016-76892-C3-3-R, TIN201567020-P and PGC2018-098813-B-C31 funded by the Spanish Government and "European Regional Development Funds (ERDF)".

${ }^{*}$ Corresponding author.

Email addresses: ad90ry@correo.ugr.es (A. Romero), jesusgonzalez@ugr .es (J. González), jamal@mcmaster.ca (M.J. Deen), tejada@ugr.es (J. A. Jiménez-Tejada)
}

which incorporate contact models and describe their currentvoltage characteristics. Overall, transistor models must be consistent with the underlying physics. In particular, the contact models must be adapted to different physical mechanisms that limit the charge injection through a metal-organic structure, such as space-charge limited conduction, thermionic emission and the Fowler-Nordheim tunneling [28, 29], or even change the properties of the contact region such as light [30,31].

A generic analytical model and associated parameter extraction method for the current-voltage characteristics of OTFTs was derived in $[19,20]$, which allows a functional model of the carrier injection through the source contact. Later works have specified this functional model to situations in which one conduction mechanism is dominant, such as the cases of spacecharge limited transport in low energy contact-barriers [21] or injection limited transport in Schottky barriers [32-35]. Despite these previous efforts, there is no standard OTFT compact model that unifies all the different physical phenomena that take place at the contact region of the OTFT. In order to choose the appropriate contact model, the expert user or decision maker (DM) should identify, in the first place, which physical phenomenon is dominant in the contact region of the OTFT. Given this lack of standardization, and in order to guide and automate the decision-making, the main objective of this work is to propose a standard contact model valid for different injection mechanisms. For that purpose, the functionality of the con- 
tact model, initially proposed in [21], is extended, resulting in a standard semi-empirical contact model. All this is detailed in Section 2.

In Section 3, the validity of the model is checked with a recently proposed multi-objective evolutionary parameter extraction method, which extracts the parameters of the model and current-voltage $\left(I_{D}-V_{C}\right)$ curves at the contact [31, 36, 37]. The results of the procedure, which analyzes output characteristics measured in transistors with the same channel length, is compared to the results of other methods based on measurements taken in transistors with different lengths. We demonstrate that, even in very-short length transistors, in which the contact resistance may be comparable to or even greater than the channel resistance, our procedure produces results comparable to methods in which a collection of transistors with short and long channels are necessary [32, 38-40]. The conclusions of the work are finally presented in Section 4.

\section{Theory}

\subsection{Models for the contact region of OTFTs}

The physical mechanisms that take place at the contact region of an OTFT influence the final functional appearance of a model that aims to describe the current-voltage characteristics of the device. This is the case of a widely-used [31, 34, 36, 41-46] generic model for OTFTs [19, 20]:

$$
\begin{aligned}
& I_{D}=k_{0} \frac{W}{L} \frac{V_{E O D R}\left(V_{G}, V_{S}\right)^{(2+\gamma)}-V_{E O D R}\left(V_{G}, V_{D}\right)^{(2+\gamma)}}{2+\gamma} \\
& k_{0}=\mu_{0} C_{O x} ; V_{E O D R}\left(V_{G}, V\right)=V_{S S} \ln \left[1+\exp \left(\frac{V_{G}-V_{T}-V}{V_{S S}}\right)\right]
\end{aligned}
$$

which includes an electric field dependent mobility

$$
\mu=\mu_{0}\left(V_{G}-V_{T}\right)^{\gamma}
$$

with $V_{T}$ being the threshold voltage, $V_{D}$ and $V_{G}$ the drain and gate terminal voltages, respectively, $W$ and $L$ the channel width and length, respectively, and $C_{O x}$ the capacitance per unit area of the gate insulator. $V_{S S}$ is a voltage parameter related to the steepness of the subthreshold characteristics of the TFT, $\mu_{0}$ is the mobility-related parameter expressed as $\mathrm{cm}^{2} /\left(\mathrm{V}^{1+\gamma} \mathrm{s}\right)$, and $\gamma=2\left(T_{0} / T-1\right)$ is the mobility enhancement factor, which is a semi-empirical parameter that depends on the temperature $T$ [47]. In order to provide a single value for the voltage dependent mobility, the mobility is evaluated at $V_{G T}=V_{G}-V_{T}=1$ $\mathrm{V}$, thus $\mu\left(V_{G T}=1 \mathrm{~V}\right)=\mu_{0}$ in $\mathrm{cm}^{2} /(\mathrm{Vs})$. This compact model is able to describe all operation modes of the transistor: triode, saturation, subthreshold or even reverse biasing.

The model (1) allows for a functional expression of the carrier injection as $V_{S} \equiv V_{C}=V_{C}\left(I_{D}, V_{G}\right)$. Different expressions have been developed in the past, depending on the dominant conduction mechanism through the contact. The cases controlled by space-charge limited transport in low energy contactbarriers [21] or injection limited transport in Schottky barriers [32-35] are reviewed below.

\subsubsection{Space-charge limited transport}

A simple model for the contact region of OTFTs in which the carrier injection is space-charge limited is [21]:

$$
\begin{gathered}
I_{D}=M_{C} \times V_{C}^{m_{k}}, \\
\forall m_{k} \in \mathbb{Z}: 1 \leq m_{k} \leq 2,
\end{gathered}
$$

where $M_{C}$ and $m_{k}$ are two parameters related to different physical variables [see (4)-(8)]. The application of the combined model (1) and (3) can be found in different kinds of OTFTs [36, 41, 48-54].

The physical origin of model (3) lies behind the solution of the transport equations in a metal-organic structure, which provides the following relation between the current density $j$ and the applied voltage $V_{C}[28,29,55,56]$ :

$$
\begin{aligned}
& V_{C}=\left(\frac{2}{3}\right)\left[\frac{2 j}{\epsilon \mu \theta}\right]^{1 / 2}\left[\left(x_{c}+x_{p}\right)^{3 / 2}-\left(x_{p}\right)^{3 / 2}\right] \\
& x_{p} \equiv \frac{j \epsilon \theta}{2 \mu[\theta q p(0)]^{2}} ; j=I_{D} / S,
\end{aligned}
$$

where $q p(0)$ is the charge density at the metal-organic interface, $\theta$ is the ratio of free to total charge density, $q \theta p$ is the free charge density, $S$ is the cross section of the channel where the drain current $I_{D}$ flows, $x_{p}$ is a characteristic length defined as the point from the contact interface towards the organic film, at which the charge density $q p\left(x_{p}\right)$ decays to $q p(0) / \sqrt{2}, \epsilon$ is the organic dielectric constant and $x_{C}$ is the length of the contact region in the organic material. Equation (4) was demonstrated to have two asymptotic trends: a linear or Ohmic behavior if the characteristic length $x_{p}$ is a few times larger than the contact length $x_{C}$

$$
I_{D} \approx \frac{S \theta q p(0) \mu}{x_{C}} V_{C} \equiv \frac{1}{R_{C}} \times V_{C}^{1},
$$

and a quadratic behavior (Mott-Gurney law) if $x_{p}$ is much smaller than $x_{C}[29,48]$

$$
I_{D} \approx \frac{9 \epsilon \theta \mu S}{8 x_{C}^{3}} V_{C}^{2} \equiv M \times V_{C}^{2}
$$

Equations (5) and (6) are the limit cases of (3) with $\left(m_{k}=1\right.$, $\left.M_{C}=1 / R_{C}\right)$ and $\left(m_{k}=2, M_{C}=M\right)$, respectively. The parameter $M_{C}$ is usually related to the gate voltage as [21]:

$$
M_{C}=\alpha\left(V_{G}-V_{T}\right)^{1+\gamma},
$$

where $\alpha$ is a proportionality constant. The subthreshold regime can be incorporated into (7) by an asymptotically interpolation function [31]

$$
M_{C}=\alpha V_{S S} \ln \left[1+\exp \left(\frac{V_{G}-V_{T}}{V_{S S}}\right)\right]^{1+\gamma} .
$$

The physical origin of this relation is found in the dependence with $\left(V_{G}-V_{T}\right)$ of both the mobility and the free charge density along the conducting channel, including the contact region [51]. The relation (7) has been checked experimentally in OTFTs that 
are free of hysteresis and free of local non-uniformities in the contact region [31, 36, 48-51]. In this regard, the relation (7) can be used as a test to detect a different behavior between the contact region and the active channel such as the existence of different kinds of traps or defects.

\subsubsection{Schottky barrier limited injection}

Different $I_{D}-V_{C}$ contact curves extracted in OTFTs resemble those of a reverse biased leaky diode. For these cases, the $I_{D}-V_{C}$ relation can be modeled as a reverse-biased Schottkydiode [32]:

$$
I_{D}=-I_{0} \exp \left[\left(\frac{V_{C}}{V_{0}}\right)^{\sigma}\right]\left\{\exp \left[-\left(\frac{V_{C}}{V_{N}}\right)\right]-1\right\},
$$

where $I_{0}$ is a gate-modulated diode-reverse-current, $V_{N}=(\eta k T) / q, q$ is the elementary charge, $\eta$ is the diode quality factor, $k$ is the Boltzmann's constant and $T$ is the absolute temperature. The barrier lowering induced by the Schottky effect is assumed in the term $\left(V_{C} / V_{0}\right)^{\sigma}$. The Schottky effect, which depends on the electric field at the junction is assumed to be dependent on the contact voltage $V_{C}$, and $V_{0}$ and $\sigma$ are fitting parameters [32]. The assumption of a contact-voltage dependent barrier lowering is also found in the study of different semiconductors such as $\mathrm{Pt} / \mathrm{Ga}_{2} \mathrm{O}_{3}$ diode contacts [57].

Despite the justified physical origin of the contact model (9) and being successfully tested in different OTFTs [32, 34, 35, 42, $58]$, the authors regard it just as a mathematical expression able to reproduce the $I_{D}-V_{C}$ curves and, consequently, the extracted diode parameters are considered as effective model parameters [33].

\subsubsection{Semi-empirical standard contact model}

The last statement of the previous section suggests that other functional expressions would also be able to reproduce the $I_{D}-V_{C}$ curves of Schottky barrier limited contacts. In this regard, and in order to incorporate different mechanisms under the same unified standard model, we propose to extend the validity of model (3) outside the range $1 \leq m_{k} \leq 2$, and include the values $0<m_{k}<1$ :

$$
\begin{gathered}
I_{D}=M_{C} \times V_{C}^{m_{k}}, \\
\forall m_{k} \in \mathbb{Z}: 0<m_{k} \leq 2 .
\end{gathered}
$$

Note that the expression (10) is a concave function if $1 \leq m_{k} \leq 2$, and a convex function if $0<m_{k}<1$. From a mathematical point of view, expression (10) with $0<m_{k}<1$ is an option to reproduce $I_{D}-V_{C}$ curves in OTFTs dominated by Schottky barriers. Note also that the use of model (10) in the range $1 \leq m_{k} \leq 2$ is physically justified as mentioned above for the space-charge dominated injection. However, since there is no physical justification for Schottky barrier dominated injection, this results in a semi-empirical model.

This is not the first time space-charge and injection limited currents in metal-organic contacts are connected under the same model. Previous numerical studies have shown that both currents can be described by exclusively solving the transport equations in the semiconductor with the only difference of specific boundary values of the free charge density at the metal-organic interface. In addition, these boundary values for the free charge density are modeled by a unified analytical expression that relates the charge density at the interface with the current density though the contact [59].

Thus, the objectives of the present work are to check the validity of this extended model (10) and to analyze the advantages of this unified model over the existing ones. The first advantage is that a single expression unifies the different mechanisms related to the injection and transport through the contacts of OTFTs. The second advantage lies behind the parameter extraction procedure that runs parallel to the compact OTFT model (1) and (10), which is introduced in the next section.

\subsection{Determination of the voltage drop at the contact}

In the past, many research efforts were made in order to quantify the contact effects of OTFTs. This is because contact effects make the extraction of the intrinsic parameters of the transistor difficult and even inaccurate. In order to determine the mobility and the threshold voltage independently of the magnitude of the contact resistance, four-probe measurements are proposed [60]. The parameter extraction techniques vary depending on whether they analyze single or multiple transistors; whether they focus on extracting the contact resistance or the voltage drop at the contact region (note that the voltage drop at the contact region is not necessarily linear, as shown in the previous sections); or whether they extract information of the contact region from current-voltage $(I-V)$ characteristics or from the voltage profile along the whole channel, as in the case of surface potentiometry [61]. Some of these procedures are reviewed in [1], including the transmission line method (TLM), which requires multiple devices with different channel lengths to determine the contact resistance [38, 39], or Impedance spectroscopy (IS) [62], which requires a single device and a proper equivalent circuit model for the OTFT in order to determine its contact resistance and evaluate the actual charge carrier mobility.

Other extraction procedures are directly linked to the generic model for OTFTs (1). One of these methods is used to determine the channel conductance of long channel devices from experimental transfer characteristics measured at low drain voltages, in which the gradual channel approximation is assumed to be valid. Then, the channel conductance is used to determine the voltage drop at the source contact in short channel devices $[32,34]$. Another parameter extraction procedure was proposed for single OTFTs operating in the linear regime, in which a proper sequence of mathematical functions derived from measured I-V characteristics are used [54].

In this work, we use a method that analyzes only the output characteristics measured in a single short-length transistor [36]. The internal parameters of the OTFT and the voltage drop at the source contact are extracted using an evolutionary procedure. The choice of this evolutionary procedure is determined firstly by its successful application to different OTFTs $[31,36,37]$. Secondly, the decision making is fully guided. 
Thus, the extraction procedure is expected to automatically detect the mechanism that dominates the injection through the contact, a Schottky barrier if $0<m_{k}<1$ or space-charge transport if $1 \leq m_{k} \leq 2$. Finally, as the parameters of the transistor can be extracted from a single device, it can compete with other well known extraction procedures that need multiple transistors with different channel lengths. In the results section, we will check that the parameters extracted from the analysis of a short-channel transistor can be used to reproduce the output characteristics measured in a long-channel device.

For the sake of simplicity and brevity, only the key facts of the evolutionary procedure defined in [36] are provided here:

(i) It is based on the model (1) and (3) to execute the parameter extraction.

(ii) The parameters of the model are extracted from output characteristics of a single OTFT.

(iii) It is automated, running in a computer from start to finish.

(iv) It is user-friendly since it does not depend on other elaborate equations.

(v) It is flexible to incorporate model modifications or new dependences of the model parameters with physical or chemical variables $[31,37]$. Just in this sense, there is no technical difference between the use of (1), with (3), as it was proposed in Ref. [36], or with (10), as used in this work, or even with (9), in case (9) would be preferred over (10). The number of parameters to be extracted would be the only difference.

\section{Results and Discussion}

In order to validate the versatility of the standard contact model (10), it is tested with transistors with Schottky-limited contacts $\left(0<m_{k}<1\right)$, space-charge-limited contacts $\left(1 \leq m_{k} \leq\right.$ $2)$, and even in transistors that when exposed to chemical treatments, have their contact region altered in such a way that any of the previous cases can be detected $\left(0<m_{k} \leq 2\right)$. In these situations, the OTFT model (1) and (10), in combination with the evolutionary procedure [36], can be seen as an effective diagnostic tool.

Four experiments $(A, B, C$ and $D$ ) have been defined, being implemented in an open source evolutionary tool called ECJ (A Java-based Evolutionary Computation Research System) [63]. This evolutionary tool has been run in a CentOS cluster with 19 computation nodes, each one with two Intel Xeon E5520 processors at $2.7 \mathrm{GHz}$. It was executed using only one node (16 threads).

\subsection{Experiments A: Schottky-limited contacts}

In this first set of experiments, we analyze the experimental output characteristics of 6,13-bis(triisopropyl-silylethynyl) (TIPS) pentacene based OTFTs [32], shown with symbols in Figs. 1a and 1b. They were measured in OTFTs with staggered top-gate configuration, with a channel width of $2000 \mu \mathrm{m}$, and with short and long-channel lengths of 10 and $200 \mu \mathrm{m}$, respectively. These OTFTs were fabricated at CEA-LITEN, using printing processes on heat stabilized, low roughness polyethylenenaphtalate PEN foils (125 $\mu \mathrm{m}$ thick). The source and drain gold contacts were defined by laser ablation. The p-type semiconductor is a solution processed TIPS-pentacene. The fluoropolymer gate dielectric $(1.2 \mu \mathrm{m}$ thick $)$ and the Ag gate electrode are both deposited by screen printing. The full protocols of the fabrication process are in [32].

The main objective of this section is to check the suitability of our model (1) and (10), in combination with the evolutionary algorithm [36], to characterize single-channel-length OTFTs with Schottky-limited contacts. To do this, we propose the following four experiments, grouped into two different evolutionary configurations: $(A l)$ the evolutionary procedure uses (1) and (10), and (A2) the procedure uses (1) and (9). First, these two configurations perform a single-transistor parameter extraction [36] (Experiments $A 1-1$ and $A 2-1$ ). They extract respective OTFT parameters from the output characteristics of the short-channel transistor shown in Fig. 1a (symbols). Subsequently, they check whether the output characteristics of the long channel transistors, shown in Fig. 1b, can be reproduced with our calculations. Second, these configurations perform a multiple-transistor parameter extraction [37] (Experiments A1-2 and A2-2). They extract respective OTFT parameters by analyzing simultaneously the output characteristics of both short- and long-channel transistors shown in Figs. 1a and 1b.

Once the experiments are executed, the values of the parameters of the models are determined. These values are collected in Table 1. They are introduced in (1) and (10) or in (1) and (9) to calculate the numerical output characteristics. Finally, they are compared to the experimental output characteristics and the Normalized Root Mean Squared Error (NRMSE [64]) are computed. The values of the NRMSE are given in Table 2 .

No significant differences are found in Table 1 for the extracted common parameters $\left(k_{0}, \gamma, V_{T}\right.$ and $\left.V_{S S}\right)$, except for $\gamma$. The values of $\gamma$ are different, but $\gamma \ll 1$. From a practical point of view $\gamma \approx 0$. It means that the mobility is almost independent on the gate voltage in the four cases. The fitting errors of the different experiments $A$ shown in Table 2 are also very similar. All these facts reinforce the similarity of the contact models (9) and (10). The error in Experiments $A 1$ is a bit lower than the error in experiments $A 2$. Also, the error when using multiple transistors is reduced, but not in a significant way. As stated in [36], the more quantity of data and time are available, the more accurate results will be reached with the evolutionary procedure.

For the sake of clarity, only the results of Experiment A1-1 are represented in Figs. 1a and $1 \mathrm{~b}$ (solid lines). Note that in this experiment $A 1-1$, the parameters of the model (1) and (10) are extracted by analyzing the experimental data of the shortchannel transistor, and subsequently used to reproduce the experimental data of the long-channel transistor. A very good agreement between the experimental and calculated data is observed in these figures. The extracted value for the carrier mobility is $\mu_{0}=1.91 \mathrm{~cm}^{2} / \mathrm{Vs}$, which is consistent with the result reported in [32], in which a value between 1.4 and $2 \mathrm{~cm}^{2} / \mathrm{Vs}$ was estimated. Also the extracted threshold voltage $V_{T}=-16$ $\mathrm{V}$ is consistent with the one given in [32], which was reported in the range -10 and $-15 \mathrm{~V}$. The $I_{D}-V_{C}$ curves extracted in this experiment are depicted with solid lines in Fig. 1c. They 
Table 1: Values of parameters extracted in Experiments $A$.

\begin{tabular}{lrrrr}
\hline & \multicolumn{4}{c}{ Experiment } \\
\cline { 2 - 5 } $\mathrm{x}$ & \multicolumn{1}{c}{$A 1-1$} & \multicolumn{1}{c}{$A 2-1$} & \multicolumn{1}{c}{$A 1-2$} & \multicolumn{1}{c}{$A 2-2$} \\
\hline$k_{0}\left[\mathrm{~A} / \mathrm{V}^{2+\gamma}\right]$ & $3.0 \times 10^{-09}$ & $2.4 \times 10^{-09}$ & $2.9 \times 10^{-09}$ & $2.3 \times 10^{-09}$ \\
$\gamma$ & $3.0 \times 10^{-03}$ & $3.9 \times 10^{-08}$ & $7.8 \times 10^{-09}$ & $7.4 \times 10^{-02}$ \\
$V_{T}[\mathrm{~V}]$ & $-1.6 \times 10^{+01}$ & $-1.9 \times 10^{+01}$ & $-1.6 \times 10^{+01}$ & $-1.7 \times 10^{+01}$ \\
$V_{S S}[\mathrm{~V}]$ & $-7.2 \times 10^{+00}$ & $-1.0 \times 10^{+01}$ & $-9.1 \times 10^{+00}$ & $-9.9 \times 10^{+00}$ \\
\hline$m_{k}$ & $4.9 \times 10^{-01}$ & & $4.7 \times 10^{-01}$ & \\
$M_{C}(-30 V)\left[\mathrm{A} / \mathrm{V}^{m_{k}}\right]$ & $4.0 \times 10^{-06}$ & & $4.1 \times 10^{-06}$ & \\
$M_{C}(-40 V)\left[\mathrm{A} / \mathrm{V}^{m_{k}}\right]$ & $9.1 \times 10^{-06}$ & & $9.6 \times 10^{-06}$ & \\
$M_{C}(-50 \mathrm{~V})\left[\mathrm{A} / \mathrm{V}^{m_{k}}\right]$ & $1.6 \times 10^{-05}$ & & $1.8 \times 10^{-05}$ & \\
\hline$V_{0}[\mathrm{~V}]$ & & $3.0 \times 10^{-01}$ & & $8.9 \times 10^{-01}$ \\
$V_{N}[\mathrm{~V}]$ & & $8.2 \times 10^{-02}$ & & $6.7 \times 10^{-01}$ \\
$\sigma$ & & $2.4 \times 10^{-01}$ & & $2.5 \times 10^{-01}$ \\
$I_{0}(-30 V)[\mathrm{A}]$ & & $1.3 \times 10^{-06}$ & & $1.9 \times 10^{-06}$ \\
$I_{0}(-40 V)[\mathrm{A}]$ & & $3.1 \times 10^{-06}$ & & $4.6 \times 10^{-06}$ \\
$I_{0}(-50 V)[\mathrm{A}]$ & & $5.3 \times 10^{-06}$ & & $8.5 \times 10^{-06}$ \\
\hline
\end{tabular}

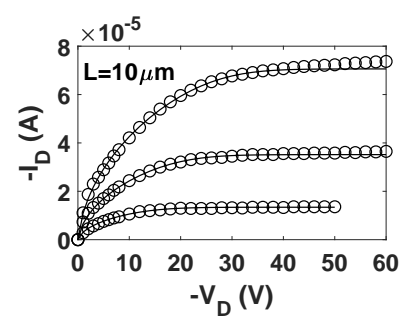

(a)

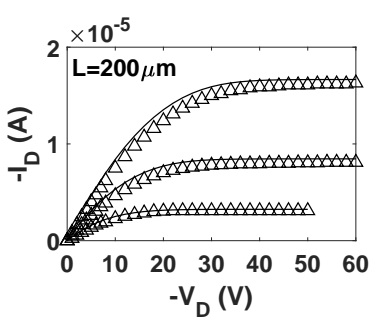

(b)

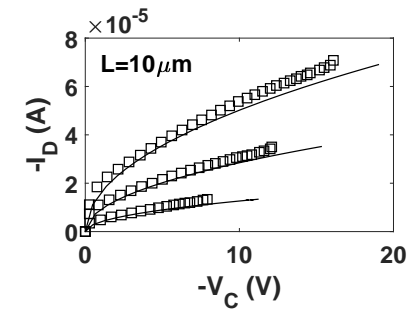

(c)

Figure 1: Experiment A1-1. (a), (b) Comparison of experimental $I_{D}-V_{D}$ curves measured at different $V_{G}$ values [32] (symbols) and our calculations with (1) and (10) (solid lines). (c) $I_{D}-V_{C}$ curves extracted with (1) and (10) at different $V_{G}$ (solid lines); the symbols show the results extracted in [32]. $V_{G}=-30,-40,-50 \mathrm{~V}$ from bottom to top.

are similar to those calculated in [32] (symbols). It is confirmed that the OTFT contact is Schottky-limited, since the evolutionary procedure converged to a parameter set with $m_{k}<1$. The dependence of $M_{C}$ with $V_{G}$ follows the trend given in (7) but using a different value of $V_{T}=V_{T}^{\prime}=-24 \mathrm{~V}$. In this case, (7) must be considered a semi-empirical relation. The reason why $M_{C}\left(V_{G}\right)$ follows (7) can be attributed to some similarities between the contact region and the active channel.

Table 2: Normalized Root Mean Squared Error (NRMSE) obtained after fitting the experimental output characteristics in Experiments $A$.

\begin{tabular}{cccc}
\hline & \multicolumn{2}{c}{ NRMSE } & Average \\
\cline { 2 - 3 } Exp. & $L=10 \mu m$ & $L=200 \mu m$ & NRMSE \\
\hline$A 1-1$ & $3.5 \times 10^{-02}$ & $1.1 \times 10^{-01}$ & $7.1 \times 10^{-02}$ \\
$A 2-1$ & $1.7 \times 10^{-02}$ & $4.3 \times 10^{-01}$ & $2.2 \times 10^{-01}$ \\
$A 1-2$ & $3.2 \times 10^{-02}$ & $3.9 \times 10^{-02}$ & $3.6 \times 10^{-02}$ \\
$A 2-2$ & $6.4 \times 10^{-02}$ & $3.0 \times 10^{-02}$ & $4.7 \times 10^{-02}$ \\
\hline
\end{tabular}

Overall, this study shows that the procedure $A l-1$ based on the analysis of a single short-channel transistor is completely equivalent to other procedures in which multiple-length transistors are needed. Most importantly, the standard model (10) is a good solution to model Schottky-limited contacts.

\subsection{Experiments B: Schottky-limited contacts}

In order to check that the previous results were not fortuitous, a new test on OTFTs with Schottky-limited contacts is carried out. In this set of experiments, we analyze the exper-

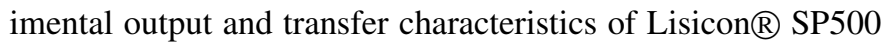
based OTFTs [35], shown with symbols in Figs. 2a-2d. They were measured in short- and long-channel OTFTs with a channel width of $1000 \mu \mathrm{m}$ and lengths of 5 and $800 \mu \mathrm{m}$, respectively.

The OTFTs were fabricated in a top-gate staggered-contact configuration with a p-type polymer. The source and drain electrodes were made of $30 \mathrm{~nm}$ thick gold patterned directly by photolithography on Gen1-size PEN foils. In order to ensure a clean surface and low contact resistance, a short oxygen 


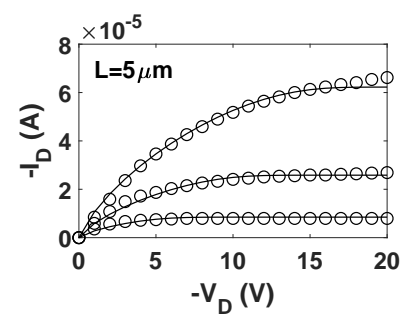

(a)

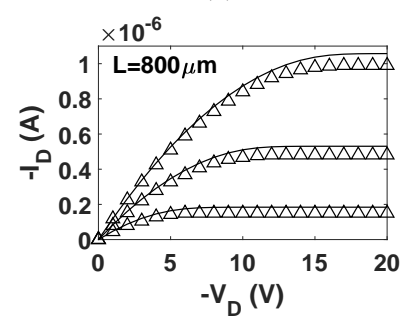

(c)

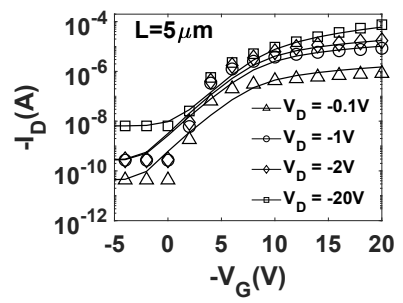

(b)

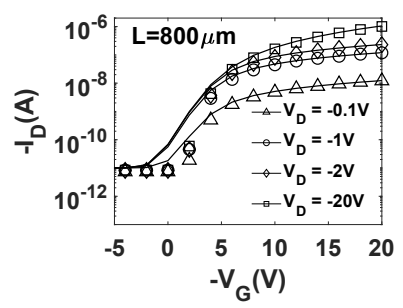

(d)

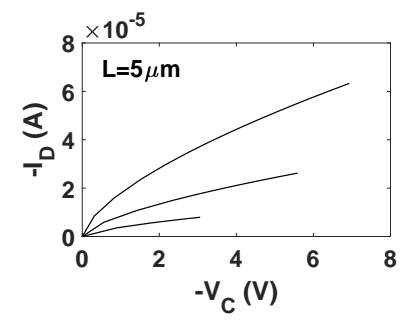

(e)

Figure 2: Experiment B1-1. (a), (c) Comparison of experimental $I_{D}-V_{D}$ curves measured at different values of $V_{G}$ [35] (symbols) and our calculations with (1) and (10) (solid lines). (b), (d) Comparison of experimental $I_{D}-V_{G}$ curves measured at different values of $V_{D}$ (symbols) and our calculations with (1) and (10) (solid lines). (e) $I_{D}-V_{C}$ curves extracted with (1) and (10) at different $V_{G}$. In (a),(c),(e), $V_{G}=-10,-15,-20 \mathrm{~V}$, from bottom to top.

plasma treatment was applied prior to the semiconductor deposition. The semiconductor thickness is in the range of $45 \mathrm{~nm}$. A

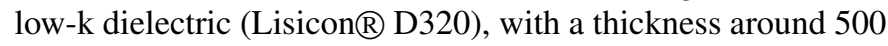
$\mathrm{nm}$ and a capacitance per unit area of $\sim 3.7 \mathrm{nF} / \mathrm{cm}^{2}$, is gravureprinted. Full details about the device fabrication are found in [35].

As in the previous case, four experiments, grouped into two different evolutionary configurations, are considered: $(B 1)$ the evolutionary procedure uses (1) and (10), and (B2) the procedure uses (1) and (9).

Experiments $B 1-1$ and $B 2-1$ perform a single-transistor parameter extraction [36]. First, they extract respective OTFT parameters from the output characteristics of the short-channel transistor shown in Fig. 2a. Then, they check whether the output characteristics of the long-channel transistor, shown in Fig. $2 \mathrm{c}$ can be reproduced with our calculations.

Experiments B1-2 and B2-2 perform a multiple-transistor parameter extraction [37]. They extract respective OTFT parameters by analyzing simultaneously the output characteristics of both short- and long-channel transistors shown in Figs. 2a and 2c.

The parameters of the transistor and the contact region, ex- tracted in all these experiments, are in Table 3. As in Experiments $A$, the values of the common parameters $\left(k_{0}, \gamma, V_{T}\right.$ and $V_{S S}$ ) for both configurations $B 1$ and $B 2$ are quite similar. The carrier mobility of these fittings is $\mu_{0}=1.6 \mathrm{~cm}^{2} / \mathrm{Vs}$, also similar to that reported in [35] $\left(2.2 \mathrm{~cm}^{2} / \mathrm{Vs}\right)$. The value of $V_{T}$ is in the range $[-4.0,-2.8] \mathrm{V}$, consistent with $V_{T}=-3.5 \mathrm{~V}$ reported in [35]. The evolutionary procedure converges to a solution with $m_{k}<1$, and $M_{C}$ follows the trend given in (7) but with a different value of $V_{T}, V_{T}^{\prime}=-7.8 \mathrm{~V}$. In the case $m_{k}<1,(7)$ is a semi-empirical relation. Nevertheless, the fact that $M_{C}\left(V_{G}\right)$ follows (7) points out that similarities between the contact region and the active channel may exist such as a uniform distribution of traps or defects along the two regions.

The values of the NRMSE of the different comparisons between the experimental output characteristics and our calculations are found in Table 4. This table shows again that: Experiment $B 1-1$ is a very valid option to characterize single shortchannel length transistors; the use of (10) or (9) is indifferent; and the use of multiple channel length transistors improves, but not significantly the fittings with the experimental data.

As an additional test, the experimental transfer characteristics of the short- and long-channel transistors shown with symbols in Figs. $2 \mathrm{~b}$ and 2d, respectively, are also fitted with our calculations. The solid lines of Figs. $2 \mathrm{a}-2 \mathrm{~d}$ show the results of Experiment $B 1-1$. The $I_{D}-V_{C}$ curves extracted in Experiment B1-1 with (10) are shown in Fig. 2e. The output and transfer characteristics and the $I_{D}-V_{C}$ curves calculated in the rest of experiments are very similar (not shown for clarity).

\subsection{Experiments $C$ : Space-charge-limited contacts}

The evolutionary parameter extraction procedure was tested previously in single channel-length OTFTs in which the injection through the contact is space-charge limited [31, 36]. Nevertheless, neither the fitting errors between experimental and calculated output characteristics of single-channel-length transistors were compared with the errors obtained if multiplechannel-length transistors are analyzed; nor this kind of contacts was assessed with a wider search space for the parameter $m_{k}$ in (10), i.e. $0 \leq m_{k} \leq 2$. In this regard, the $I-V$ characteristics of multiple-length, bottom-gate, bottom-contact TFTs, with dinaphtho[2,3-b:20,30-f] thieno[3,2-b]thiophene (DNTT) as semiconductor, are studied. They were measured in [40], and the measurement data are represented with symbols in Fig. 3. The Au contacts of the transistors were functionalized with pentafluorobenzenethiol (PFBT) to obtain a small contact resistance. All TFTs were fabricated on flexible plastic using aluminum oxide $\left(\mathrm{Al}_{2} \mathrm{O}_{3}\right)$ as the gate dielectric $(5.3 \mathrm{~nm}$ thick). The full protocols of the electrode deposition, substrate cleaning, and surface passivation are in [40, 65]. Short and longchannel TFTs were fabricated with a channel width of $200 \mu \mathrm{m}$ and lengths of 8,40 and $80 \mu \mathrm{m}$.

The evolutionary procedure is used only with (1) and (10), since model (9) is no longer valid in these cases. Three experiments $C-1, C-2$ and $C-3$ are designed. Experiment $C-1$ is performed for a single-transistor parameter extraction. It extracts the parameters of the transistor and the contact region from the output characteristics of the shortest transistor (circles 
Table 3: Values of parameters extracted in Experiments $B$.

\begin{tabular}{lrrrr}
\hline & \multicolumn{4}{c}{ Experiment } \\
\cline { 2 - 5 } $\mathrm{x}$ & \multicolumn{1}{c}{$B 1-1$} & \multicolumn{1}{c}{$B 2-1$} & \multicolumn{1}{c}{$B 1-2$} & \multicolumn{1}{c}{$B 2-2$} \\
\hline$k_{0}\left[\mathrm{~A} / \mathrm{V}^{2+\gamma}\right]$ & $5.8 \times 10^{-09}$ & $5.8 \times 10^{-09}$ & $5.9 \times 10^{-09}$ & $5.7 \times 10^{-09}$ \\
$\gamma$ & $3.5 \times 10^{-10}$ & $1.4 \times 10^{-02}$ & $3.8 \times 10^{-03}$ & $1.5 \times 10^{-02}$ \\
$V_{T}[\mathrm{~V}]$ & $-2.8 \times 10^{+00}$ & $-4.0 \times 10^{+00}$ & $-3.6 \times 10^{+00}$ & $-3.4 \times 10^{+00}$ \\
$V_{S S}[\mathrm{~V}]$ & $-1.3 \times 10^{+00}$ & $-2.0 \times 10^{+00}$ & $-1.4 \times 10^{+00}$ & $-1.3 \times 10^{+00}$ \\
\hline$m_{k}$ & $6.5 \times 10^{-01}$ & & $7.1 \times 10^{-01}$ & \\
$M_{C}(-10 \mathrm{~V})\left[\mathrm{A} / \mathrm{V}^{m_{k}}\right]$ & $3.8 \times 10^{-06}$ & & $3.9 \times 10^{-06}$ & \\
$M_{C}(-15 \mathrm{~V})\left[\mathrm{A} / \mathrm{V}^{m_{k}}\right]$ & $8.6 \times 10^{-06}$ & & $8.3 \times 10^{-06}$ & \\
$M_{C}(-20 \mathrm{~V})\left[\mathrm{A} / \mathrm{V}^{m_{k}}\right]$ & $1.8 \times 10^{-05}$ & & $1.7 \times 10^{-05}$ & \\
\hline$V_{0}$ & & $7.1 \times 10^{-01}$ & & $9.9 \times 10^{-01}$ \\
$V_{N}$ & & $3.2 \times 10^{-03}$ & & $9.9 \times 10^{-01}$ \\
$\sigma$ & & $4.1 \times 10^{-01}$ & & $4.6 \times 10^{-01}$ \\
$I_{0}(-10 \mathrm{~V})[\mathrm{A}]$ & & $1.6 \times 10^{-06}$ & & $1.7 \times 10^{-06}$ \\
$I_{0}(-15 V)[\mathrm{A}]$ & & $3.1 \times 10^{-06}$ & & $3.5 \times 10^{-06}$ \\
$I_{0}(-20 \mathrm{~V})[\mathrm{A}]$ & & $6.0 \times 10^{-06}$ & & $6.4 \times 10^{-06}$ \\
\hline
\end{tabular}

Table 4: Normalized Root Mean Squared Error (NRMSE) obtained after fitting the experimental output characteristics in Experiments $B$.

\begin{tabular}{cccc}
\hline & \multicolumn{2}{c}{ NRMSE } & Average \\
\cline { 2 - 3 } Sub-exp. & $L=5 \mu m$ & $L=800 \mu m$ & NRMSE \\
\hline$B 1-1$ & $5.1 \times 10^{-02}$ & $1.2 \times 10^{-01}$ & $8.5 \times 10^{-02}$ \\
$B 2-1$ & $5.4 \times 10^{-02}$ & $7.8 \times 10^{-02}$ & $6.6 \times 10^{-02}$ \\
$B 1-2$ & $5.6 \times 10^{-02}$ & $3.1 \times 10^{-02}$ & $4.4 \times 10^{-02}$ \\
$B 2-2$ & $6.2 \times 10^{-02}$ & $7.3 \times 10^{-03}$ & $3.5 \times 10^{-02}$ \\
\hline
\end{tabular}

in Fig. 3a). Then, it checks whether the output characteristics of the two longest transistors are reproduced with our calculations. Experiments $C$ - 2 and $C$ - 3 perform a multiple-transistor parameter extraction. Experiment $C$ - 2 extracts the model parameters from the simultaneous analysis of the experimental data of the shortest and longest transistors (symbols in Figs. 3a and 3e, respectively). Then, it checks whether the output characteristics of the $40 \mu \mathrm{m}$ length transistor (symbols in Fig. 3c) are reproduced with our calculations. Experiment $C-3$ extracts the model parameters from the simultaneous analysis of the experimental data (symbols) of Figs. 3a, 3c and 3e.

The values of the parameters extracted with the three experiments are found in Table 5. Very small variations are observed among the experiments. The carrier mobility extracted with Experiment $C-1$ is $\mu_{0}=2.9 \mathrm{~cm}^{2} / \mathrm{Vs}$, and the threshold voltage is $V_{T}=-1.2 \mathrm{~V}$, in agreement with the respective values extracted with the TLM method in [40]: $3.2 \mathrm{~cm}^{2} / \mathrm{Vs}$ and $-1.3 \mathrm{~V}$. The value $m_{k}>1$ points out that the injection in these TFTs is space-charge limited. In these Experiments $C, M_{C}\left(V_{G}\right)$ does not follow (7), suggesting the existence of local non-uniformities close to the contact region. A linear interpolation function is built in order to determine the value of $M_{C}$ at any other value of $V_{G}$. The errors obtained when fitting the experimental and calculated output characteristics are in Table 6. The fitting error is slightly reduced when the amount of available data increases (from Experiment $C-1$ to $C-3$ ). However, the improvement is not significant. As in the previous experiments, only the $I-V$ curves calculated with the single-transistor procedure (Experiment $C-1$ ) are represented in Fig. 3 (solid lines). As an additional test, this figure includes the comparison of experimental (symbols) and calculated (solid lines) transfer characteristics, also showing a very good agreement.

\subsection{Experiment D: Application to an ammonia sensor}

The standard contact model (10) takes on a special meaning in situations in which both the active channel and the contact region are affected by an external excitation, and both jointly determine the electrical behavior of the device. In this sense, we apply the evolutionary procedure to an OTFT detector of ammonia $\left(\mathrm{NH}_{3}\right)$ gas [66]. In this publication, authors checked that the ammonia sensor deteriorated its electrical performance under the exposure of different concentrations of ammonia. Here, we check that the incorporation of a standard contact model (10) in the compact TFT model (1), not only enhances the precision in the determination of the intrinsic parameters of the transistor, but also provides information on how the contact region itself reacts to the ammonia gas. Our evolutionary procedure is applied to the output characteristics of a regioregular poly (3hexylthiophene) (rr-P3HT) based OFET, which are represented with symbols in Figs. 4a (pristine device), 4c (gas exposure of $20 \mathrm{ppm}$ ) and $4 \mathrm{e}$ (gas exposure of $40 \mathrm{ppm}$ ) [66]. 
Table 5: Values of parameters extracted in Experiments $C$.

\begin{tabular}{lrrr}
\hline & \multicolumn{3}{c}{ Experiment } \\
\cline { 2 - 4 } $\mathrm{x}$ & \multicolumn{1}{c}{$C 1-1$} & \multicolumn{1}{c}{$C 1-2$} & \multicolumn{1}{c}{$C 1-3$} \\
\hline$k_{0}\left[\mathrm{~A} / \mathrm{V}^{2+\gamma}\right]$ & $2.0 \times 10^{-06}$ & $2.2 \times 10^{-06}$ & $1.5 \times 10^{-06}$ \\
$\gamma$ & $5.0 \times 10^{-01}$ & $6.6 \times 10^{-02}$ & $4.0 \times 10^{-01}$ \\
$V_{T}[\mathrm{~V}]$ & $-1.2 \times 10^{+00}$ & $-1.1 \times 10^{+00}$ & $-9.3 \times 10^{-01}$ \\
$V_{S S}[\mathrm{~V}]$ & $-6.7 \times 10^{-01}$ & $-2.9 \times 10^{-01}$ & $-1.2 \times 10^{-01}$ \\
$m_{k}$ & $1.6 \times 10^{+00}$ & $1.3 \times 10^{+00}$ & $1.4 \times 10^{+00}$ \\
$M_{C}(-1.67 \mathrm{~V})\left[\mathrm{A} / \mathrm{V}^{m_{k}}\right]$ & $2.0 \times 10^{-04}$ & $6.7 \times 10^{-05}$ & $8.6 \times 10^{-05}$ \\
$M_{C}(-2.00 \mathrm{~V})\left[\mathrm{A} / \mathrm{V}^{m_{k}}\right]$ & $3.7 \times 10^{-04}$ & $1.2 \times 10^{-04}$ & $2.0 \times 10^{-04}$ \\
$M_{C}(-2.33 \mathrm{~V})\left[\mathrm{A} / \mathrm{V}^{m_{k}}\right]$ & $3.7 \times 10^{-04}$ & $1.5 \times 10^{-04}$ & $2.5 \times 10^{-04}$ \\
$M_{C}(-2.67 \mathrm{~V})\left[\mathrm{A} / \mathrm{V}^{m_{k}}\right]$ & $2.7 \times 10^{-04}$ & $1.5 \times 10^{-04}$ & $2.4 \times 10^{-04}$ \\
$M_{C}(-3.00 \mathrm{~V})\left[\mathrm{A} / \mathrm{V}^{m_{k}}\right]$ & $1.8 \times 10^{-04}$ & $1.2 \times 10^{-04}$ & $1.5 \times 10^{-04}$ \\
\hline
\end{tabular}

Table 6: Normalized Root Mean Squared Error (NRMSE) obtained after fitting the experimental output characteristics in Experiments $C$.

\begin{tabular}{ccccc}
\hline & \multicolumn{3}{c}{ NRMSE } & Average \\
\cline { 2 - 4 } Exp. & $L=8 \mu m$ & $L=40 \mu m$ & $L=80 \mu m$ & NRMSE \\
\hline$C-1$ & $7.8 \times 10^{-02}$ & $8.7 \times 10^{-02}$ & $1.2 \times 10^{-01}$ & $9.5 \times 10^{-02}$ \\
$C-2$ & $3.4 \times 10^{-02}$ & $1.3 \times 10^{-01}$ & $5.8 \times 10^{-02}$ & $7.4 \times 10^{-02}$ \\
$C-3$ & $5.3 \times 10^{-02}$ & $5.7 \times 10^{-02}$ & $7.6 \times 10^{-02}$ & $6.2 \times 10^{-02}$ \\
\hline
\end{tabular}

The full fabrication protocols of the rr-P3HT OTFT are detailed in [66]. Briefly, the bottom-gate bottom-contact OFET consists of a heavily doped p-type Si substrate acting as the gate structure, and a $300 \mathrm{~nm}$ thick film of thermally grown $\mathrm{SiO}_{2}$ on top of it. The capacitance per unit area of the gate dielectric layer is $10 \mathrm{nF} / \mathrm{cm}^{2}$. The gold source-drain electrodes and the rr-P3HT polymer define a channel length and a width of $30 \mu \mathrm{m}$ and $1 \mathrm{~mm}$, respectively. Information about the electrode deposition, substrate cleaning and surface passivation is found in [66].

Since the gas exposure changes the electrical performance of the device, the evolutionary procedure must treat independently each set of experimental $I_{D}-V_{D}$ curves (symbols in Figs. $4 \mathrm{a}, 4 \mathrm{c}$ and $4 \mathrm{e}$ ). The values of the OTFT parameters, extracted in each of the three cases, are in Table 7 . The $I_{D}-V_{D}$ curves calculated with (1) and (10) are depicted with solid lines in Figs. 4a, 4c and $4 \mathrm{e}$, where a good agreement with the experimental data is shown. The $I_{D}-V_{C}$ curves extracted with our procedure for the respective three cases are depicted in Figs. 4b, $4 \mathrm{~d}$ and $4 \mathrm{f}$.

The exposure to gas ammonia changes the value of the carrier mobility and the threshold voltage of the transistor, as observed in [66]. However, the values of these parameters largely differ because, in our case, the contacts effects are considered during the extraction process. With our evolutionary procedure, $V_{T}$ varies in the range 7.65 and $-12.7 \mathrm{~V}$, for the pristine OTFT and for the $40 \mathrm{ppm}$ gas exposure case, respectively; while the values of $\mu_{0}$ are $2.9 \times 10^{-4}, 8.2 \times 10^{-5}$ and $6.3 \times 10^{-5}$ for the pristine
OTFT and under a exposure of 20 and $40 \mathrm{ppm}$, respectively.

The main contribution of this analysis is the evolution of the current-voltage curves at the contact when the concentration of ammonia gas increases. Fig. $4 \mathrm{~b}$ shows the $I_{D}-V_{C}$ curves extracted for the pristine OTFT at different values of $V_{G}$. The curves are concave following (10) with $m_{k}=1.21$. Figs. $4 \mathrm{~d}$ and $4 \mathrm{f}$ show the $I_{D}-V_{C}$ curves extracted for the exposed OTFT under ammonia gas at different values of $V_{G}$. These two sets of curves become convex, also following (10) but with $m_{k}<1$ $\left(m_{k}=0.69\right.$ and $m_{k}=0.57$ for gas concentrations of 20 and $40 \mathrm{ppm}$, respectively). A clear deterioration of the contact region is observed when the ammonia concentration is increased. This can be attributed to chemical changes at the metal-organic interface that alter the energy barrier that charge carriers must overcome. These changes drastically modify the contact region from a space-charge to a Schottky barrier limited contact. This deterioration is also visible in the $M_{C}\left(V_{G}\right)$ relation extracted in the three cases. None of the extracted $M_{C}$ sets from Table 7 fulfills (7), which again can be explained by non-uniformities allocated between the active channel and the contact region, that deteriorate by increasing the gas concentration.

\section{Conclusions}

We have proposed a versatile analytical expression that can reproduce the current-voltage curves at the contacts of OTFTs. The expression is controlled exclusively with two parameters. 
Table 7: Values of parameters extracted in Experiments $D$.

\begin{tabular}{lrrr}
\hline & \multicolumn{3}{c}{ Ammonia Concentration $(\mathrm{ppm})$} \\
\cline { 2 - 4 } $\mathrm{x}$ & \multicolumn{1}{c}{0} & \multicolumn{1}{c}{20} & \multicolumn{1}{c}{40} \\
\hline$k_{0}\left[\mathrm{~A} / \mathrm{V}^{2+\gamma}\right]$ & $2.9 \times 10^{-12}$ & $8.2 \times 10^{-13}$ & $6.3 \times 10^{-13}$ \\
$\gamma$ & $2.9 \times 10^{-01}$ & $8.3 \times 10^{-01}$ & $1.1 \times 10^{-00}$ \\
$V_{T}[\mathrm{~V}]$ & $3.0 \times 10^{+01}$ & $7.7 \times 10^{+00}$ & $-1.3 \times 10^{+01}$ \\
$V_{S S}[\mathrm{~V}]$ & $-1.5 \times 10^{+01}$ & $-3.2 \times 10^{+01}$ & $-3.1 \times 10^{+01}$ \\
$m_{k}$ & $1.2 \times 10^{+00}$ & $6.9 \times 10^{-01}$ & $5.7 \times 10^{-01}$ \\
$M_{C}(-10 \mathrm{~V})\left[\mathrm{A} / \mathrm{V}^{m_{k}}\right]$ & $1.8 \times 10^{-07}$ & $5.4 \times 10^{-08}$ & $2.7 \times 10^{-08}$ \\
$M_{C}(-20 \mathrm{~V})\left[\mathrm{A} / \mathrm{V}^{m_{k}}\right]$ & $4.5 \times 10^{-08}$ & $5.3 \times 10^{-08}$ & $4.2 \times 10^{-08}$ \\
$M_{C}(-30 \mathrm{~V})\left[\mathrm{A} / \mathrm{V}^{m_{k}}\right]$ & $2.4 \times 10^{-08}$ & $5.0 \times 10^{-08}$ & $5.1 \times 10^{-08}$ \\
$M_{C}(-40 \mathrm{~V})\left[\mathrm{A} / \mathrm{V}^{m_{k}}\right]$ & $1.7 \times 10^{-08}$ & $5.5 \times 10^{-08}$ & $4.7 \times 10^{-08}$ \\
\hline & & & \\
\hline
\end{tabular}

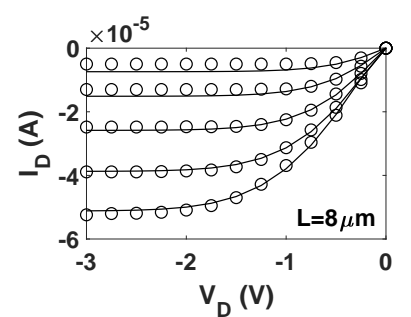

(a)

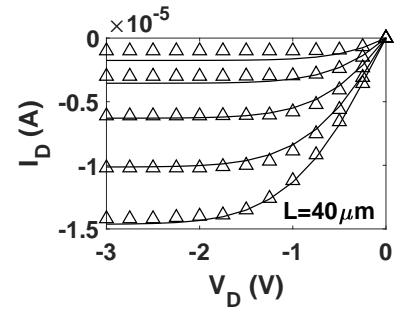

(c)

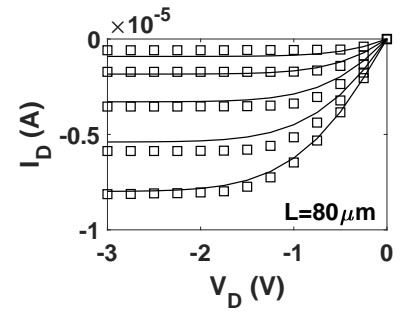

(e)

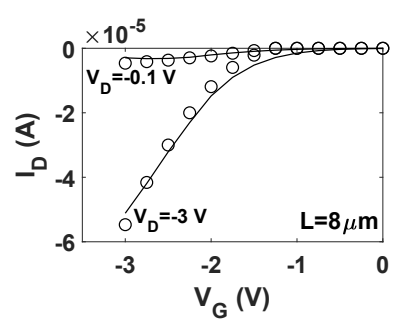

(b)

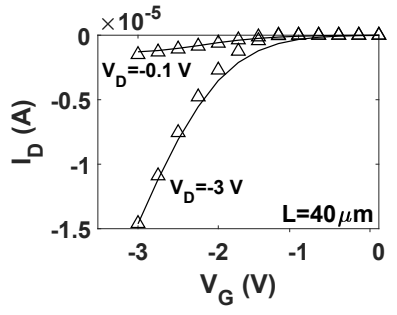

(d)

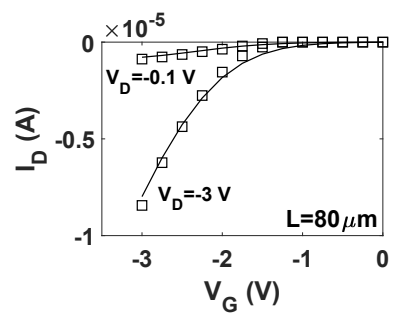

(f)

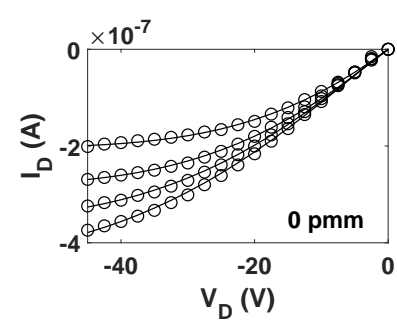

(a)

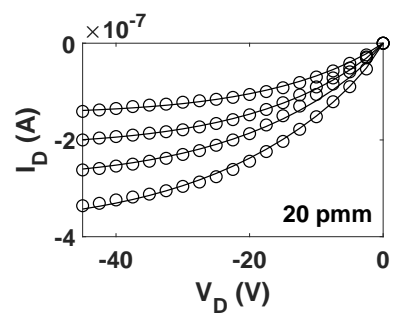

(c)

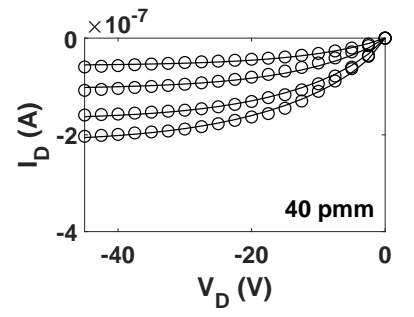

(e)

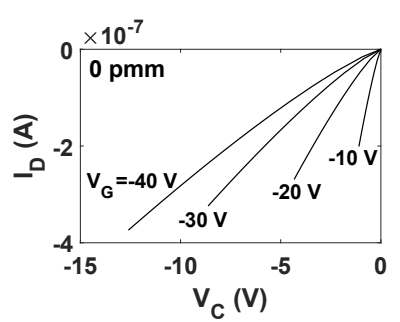

(b)

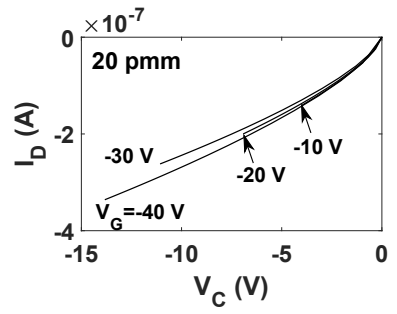

(d)

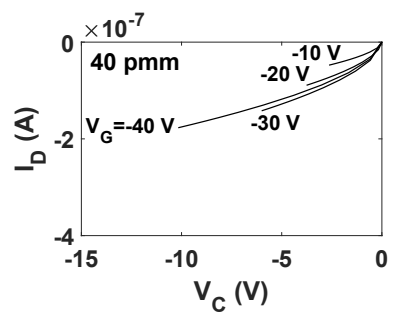

(f)
Figure 3: Experiment $C$-1. (a), (c), (e) Comparison of experimental $I_{D}-V_{D}$ curves measured at different values of $V_{G}$ [40] (symbols) and our calculations with (1) and (10) (solid lines). $V_{G}$ is swept from -1.67 (top) to $-3 \mathrm{~V}$ (bottom) with a $-0.33 \mathrm{~V}$ step. (b), (d), (f) Comparison of experimental $I_{D}-V_{G}$ curves measured at different values of $V_{D}$ [40] (symbols) and our calculations with (1) and (10) (solid lines).

One of them depends on the gate voltage. The other parameter allows to distinguish between space-charge or Schottky limited contacts. The contact model has a direct physical justification when space-charge limited contacts are treated. In the case
Figure 4: Experiment D. (a), (c), (e) Comparison of experimental $I_{D}-V_{D}$ curves (symbols) and our calculations with (1) and (10) (solid lines) at three concentrations of ammonia and different $V_{G}$ values. $V_{G}$ is swept from -10 (top) to $-40 \mathrm{~V}$ (bottom) with a $-10 \mathrm{~V}$ step. (b), (d), (f) $I_{D}-V_{C}$ curves calculated with (10) at different $V_{G}$ values.

of Schottky-limited contacts, our model is empirical, although it has been successfully compared with other semi-empirical models based on the current transport theory in metal semiconductor barriers.

The contact model is introduced in a generic compact model 
that describes the current-voltage characteristics of OTFTs. The contact model used in combination with an evolutionary parameter extraction method allows to extract the parameters of short channel OTFTs. We have checked that our procedure obtains similar results to those methods in which multiple transistors with different lengths are necessary in order to characterize the effects of the contact region.

The procedure has been also applied to an ammonia gas sensor in order to determine the effect of the gas, not only in the internal parameters of the transistor, carrier mobility or threshold voltage, but also in the contact region. We have detected that the gas can modify the energy barrier at the contact in such a way that a space-charge limited contact is transformed into a Schottky-limited contact when ammonia gas is present.

\section{References}

[1] Z. A. Lamport, H. F. Haneef, S. Anand, M. Waldrip, O. D. Jurchescu, Tutorial: Organic field-effect transistors: Materials, structure and operation, J. Appl. Phys. 124 (7) (2018) 071101.

[2] X. Guo, Y. Xu, S. Ogier, T.-N. Ng, M. Caironi, A. Perinot, L. Li, J. Zhao, W. Tang, R. A. Sporea, A. Nejim, J. Carrabina, P. Cain, F. Yan, Current status and opportunities of organic thin-film transistor technologies, IEEE Trans. Electron Devices 64 (5) (2017) 1906-1921.

[3] C.-J. Lee, Y.-C. Chang, L.-W. Wang, Y.-H. Wang, Biodegradable materials for organic field-effect transistors on a paper substrate, IEEE Electron Device Lett. 40 (2) (2019) 236-239.

[4] M. Kondo, T. Uemura, M. Akiyama, N. Namba, M. Sugiyama, Y. Noda, T. Araki, S. Yoshimoto, T. Sekitani, Design of ultraflexible organic differential amplifier circuits for wearable sensor technologies, in: 2018 IEEE International Conference on Microelectronic Test Structures (ICMTS), IEEE, 2018.

[5] Y. Yuan, G. Giri, A. L. Ayzner, A. P. Zoombelt, S. C. Mannsfeld, J. Chen, D. Nordlund, M. F. Toney, J. Huang, Z. Bao, Ultra-high mobility transparent organic thin film transistors grown by an off-centre spin-coating method, Nat. Commun. 5 (3005) (2014) 1-9.

[6] X. Xu, Y. Yao, B. Shan, X. Gu, D. Liu, J. Liu, J. Xu, N. Zhao, W. Hu, Q. Miao, Electron mobility exceeding $10 \mathrm{~cm}^{2} \mathrm{v}^{-1} \mathrm{~s}^{-1}$ and band-like charge transport in solution-processed n-channel organic thin-film transistors, Adv. Mater. 28 (26) (2016) 5276-5283.

[7] A. F. Paterson, N. D. Treat, W. Zhang, Z. Fei, G. Wyatt-Moon, H. Faber, G. Vourlias, P. A. Patsalas, O. Solomeshch, N. Tessler, M. Heeney, T. D. Anthopoulos, Small molecule/polymer blend organic transistors with hole mobility exceeding $13 \mathrm{~cm}^{2} / \mathrm{Vs}$, Adv. Mater. 28 (35) (2016) 7791-7798.

[8] D. He, J. Qiao, L. Zhang, J. Wang, T. Lan, J. Qian, Y. Li, Y. Shi, Y. Chai, W. Lan, L. K. Ono, Y. Qi, J.-B. Xu, W. Ji, X. Wang, Ultrahigh mobility and efficient charge injection in monolayer organic thin-film transistors on boron nitride, Science Advances 3 (9).

[9] F. Rasheed, M. S. Golanbari, G. C. Marques, M. B. Tahoori, J. AghassiHagmann, A smooth EKV-based DC model for accurate simulation of printed transistors and their process variations, IEEE Trans. Electron Devices 65 (2) (2018) 667-673.

[10] X. Li, G. S. Lee, S. H. Park, H. Kong, T. K. An, S. H. Kim, Direct writing of silver nanowire electrodes via dragging mode electrohydrodynamic jet printing for organic thin film transistors, Org. Electron. 62 (2018) 357 365.

[11] R. A. Street, A. Salleo, Contact effects in polymer transistors, Appl. Phys. Lett. 81 (15) (2002) 2887-2889.

[12] M. Marinkovic, D. Belaineh, V. Wagner, D. Knipp, On the origin of contact resistances of organic thin film transistors, Adv. Mater. 24 (29) (2012) 4005-4009.

[13] S. Choi, C. Fuentes-Hernandez, C.-Y. Wang, T. M. Khan, F. A. Larrain, Y. Zhang, S. Barlow, S. R. Marder, B. Kippelen, A study on reducing contact resistance in solution-processed organic field-effect transistors, ACS Appl. Mater. Inter. 8 (37) (2016) 24744-24752.

[14] D. Natali, M. Caironi, Charge injection in solution-processed organic field-effect transistors: Physics, models and characterization methods, Adv. Mater. 24 (11) (2012) 1357-1387.

[15] C. Liu, G. Li, R. D. Pietro, J. Huang, Y.-Y. Noh, X. Liu, T. Minari, Device physics of contact issues for the overestimation and underestimation of carrier mobility in field-effect transistors, Phys. Rev. Appl. 8 (3).

[16] Z. A. Lamport, K. J. Barth, H. Lee, E. Gann, S. Engmann, H. Chen, M. Guthold, I. McCulloch, J. E. Anthony, L. J. Richter, D. M. DeLongchamp, O. D. Jurchescu, A simple and robust approach to reducing contact resistance in organic transistors, Nat. Commun. 9 (1).

[17] L. Mariucci, M. Rapisarda, A. Valletta, G. Fortunato, Contact effects in organic thin-film transistors: Device physics and modeling, in: Handbook of Visual Display Technology, Springer International Publishing, 2016, pp. 945-969.

[18] S. D. Wang, Y. Yan, K. Tsukagoshi, Understanding contact behavior in organic thin film transistors, Appl. Phys. Lett. 97 (6) (2010) 063307.

[19] O. Marinov, M. J. Deen, U. Zschieschang, H. Klauk, Organic thin-film transistors: Part I-compact dc modeling, IEEE Trans. Electron Devices 56 (2009) 2952-2961.

[20] M. J. Deen, O. Marinov, U. Zschieschang, H. Klauk, Organic Thin-Film Transistors: Part II. Parameter Extraction, IEEE Trans. Electron Devices 56 (12) (2009) $2962-2968$.

[21] J. A. Jiménez-Tejada, J. A. López-Villanueva, P. López-Varo, K. M. Awawdeh, M. J. Deen, Compact modeling and contact effects in organic transistors, IEEE Trans. Electron Devices 61 (2) (2014) 266-277.

[22] B. Li, C. Y. Han, P. T. Lai, W. M. Tang, Effects of source/drain-electrode material, thickness and fabrication method on the electrical performance of pentacene thin-film transistor, Thin Solid Films 667 (2018) 28 - 33.

[23] S. Nair, M. Kathiresan, T. Mukundan, Two dimensional simulation of patternable conducting polymer electrode based organic thin film transistor, Semicond. Sci. and Tech. 33 (2) (2018) 025006.

[24] T. Borthakur, R. Sarma, Top-contact pentacene-based organic thin film transistor (OTFT) with n,n'-bis(3-methyl phenyl)-n,n'-diphenyl benzidine (TPD)/au bilayer source-drain electrode, J. Electron. Mater. 47 (1) (2018) 627-632.

[25] Y. Mei, D. Fogel, J. Chen, J. W. Ward, M. M. Payne, J. E. Anthony, O. D. Jurchescu, Interface engineering to enhance charge injection and transport in solution-deposited organic transistors, Org. Electron. 50 (2017) 100 105.

[26] A. F. Paterson, Y.-H. Lin, A. D. Mottram, Z. Fei, M. R. Niazi, A. R. Kirmani, A. Amassian, O. Solomeshch, N. Tessler, M. Heeney, T. D. Anthopoulos, The impact of molecular p-doping on charge transport in highmobility small-molecule/polymer blend organic transistors, Adv. Electron. Mater. 4 (10) (2018) 1700464.

[27] C.-H. Kim, G. Horowitz, Toward a fully analytical contact resistance expression in organic transistors, Materials 12 (7).

[28] P. Lara Bullejos, J. A. Jiménez-Tejada, M. J. Deen, O. Marinov, W. R. Datars, Unified model for the injection and transport of charge in organic diodes, J. Appl. Phys. 103 (6) (2008) 064504.

[29] P. Lara Bullejos, J. A. Jiménez-Tejada, S. Rodríguez-Bolívar, M. J. Deen, O. Marinov, Model for the injection of charge through the contacts of organic transistors, J. Appl. Phys. 105 (8) (2009) 084516.

[30] M. Deen, M. Kazemeini, Photosensitive polymer thin-film FETs based on poly(3-octylthiophene), Proceedings of the IEEE 93 (7) (2005) 13121320.

[31] A. Romero, C. Jiménez, J. González, P. López-Varo, M. J. Deen, J. A. Jiménez-Tejada, Compact modeling of the effects of illumination on the contact region of organic phototransistors, Org. Electron. 70 (2019) 113 $-121$.

[32] A. Valletta, A. Daami, M. Benwadih, R. Coppard, G. Fortunato, M. Rapisarda, F. Torricelli, L. Mariucci, Contact effects in high performance fully printed p-channel organic thin film transistors, Appl. Phys. Lett. 99 (23) (2011) 233309.

[33] L. Mariucci, M. Rapisarda, A. Valletta, S. Jacob, M. Benwadih, G. Fortunato, Current spreading effects in fully printed p-channel organic thin film transistors with schottky source-drain contacts, Org. Electron. 14 (1) (2013) 86-93.

[34] A. Valletta, M. Rapisarda, S. Calvi, G. Fortunato, M. Frasca, G. Maira, A. Ciccazzo, L. Mariucci, A DC and small signal AC model for organic thin film transistors including contact effects and non quasi static regime, Org. Electron. 41 (Supplement C) (2017) 345-354.

[35] M. Charbonneau, D. Locatelli, S. Lombard, C. Serbutoviez, L. Tournon, 
F. Torricelli, S. Abdinia, E. Cantatore, M. Fattori, A large-area gravure printed process for p-type organic thin-film transistors on plastic substrates, in: 2018 48th European Solid-State Device Research Conference (ESSDERC), IEEE, 2018.

[36] A. Romero, J. González, R. Picos, M. J. Deen, J. A. Jiménez-Tejada, Evolutionary parameter extraction for an organic tft compact model including contact effects, Org. Electron. 61 (2018) $242-253$

[37] A. Romero, J. González, J. A. Jimenez-Tejada, Constrained manyobjective evolutionary extraction procedure for an OTFT compact model including contact effects, in: 2018 Spanish Conference on Electron Devices (CDE), IEEE, 2018.

[38] H. H. Berger, Contact resistance and contact resistivity, J. Electrochem. Soc. 119 (4) (1972) 507-514.

[39] U. Kraft, J. E. Anthony, E. Ripaud, M. A. Loth, E. Weber, H. Klauk, Lowvoltage organic transistors based on tetraceno[2,3-b]thiophene: Contact resistance and air stability, Chem. Mater. 27 (3) (2015) 998-1004.

[40] M. Krammer, J. Borchert, A. Petritz, E. Karner-Petritz, G. Schider, B. Stadlober, H. Klauk, K. Zojer, Critical evaluation of organic thin-film transistor models, Crystals 9 (2) (2019) 85.

[41] M. Fayez, K. M. Morsi, M. N. Sabry, OTFTs compact models: analysis, comparison, and insights, IET Circ. Device. Syst. 11 (5) (2017) 409-420.

[42] S. Abdinia, F. Torricelli, G. Maiellaro, R. Coppard, A. Daami, S. Jacob, L. Mariucci, G. Palmisano, E. Ragonese, F. Tramontana, A. van Roermund, E. Cantatore, Variation-based design of an AM demodulator in a printed complementary organic technology, Org. Electron. 15 (4) (2014) 904-912.

[43] A. M. Ma, D. W. Barlage, Analysis of the channel and contact regions in staggered and drain-offset $\mathrm{ZnO}$ thin-film transistors with compact modeling, IEEE Trans. Electron Devices 65 (8) (2018) 3277-3282.

[44] R. Martins, D. Gaspar, M. J. Mendes, L. Pereira, J. Martins, P. Bahubalindruni, P. Barquinha, E. Fortunato, Papertronics: Multigate paper transistor for multifunction applications, Appl. Mater. Today 12 (2018) 402-414.

[45] M. Buonomo, N. Lago, G. Cantarella, N. Wrachien, M. Natali, F. Prescimone, E. Benvenuti, M. Muccini, S. Toffanin, A. Cester, Simple and accurate single transistor technique for parameters extraction from organic and inorganic thin film devices, Org. Electron. 63 (2018) 376-383.

[46] S. Fatima, U. Rafique, U. Ahmed, M. Ahmed, A global parameters extraction technique to model organic field effect transistors output characteristics, Solid-State Electron. 152 (2019) 81-92.

[47] O. Marinov, M. J. Deen, R. Datars, Compact modeling of charge carrier mobility in organic thin-film transistors, J. Appl. Phys. 106 (6) (2009) 064501 .

[48] J. A. Jiménez-Tejada, K. M. Awawdeh, J. A. López-Villanueva, J. E. Carceller, M. J. Deen, N. B. Chaure, T. Basova, A. K. Ray, Contact effects in compact models of organic thin film transistors: Application to zinc phthalocyanine-based transistors, Org. Electron. 12 (5) (2011) 832-842.

[49] J. A. Jiménez-Tejada, P. López-Varo, A. N. Cammidge, I. Chambrier, M. J. Cook, N. B. Chaure, A. K. Ray, Compact modeling of organic thinfilm transistors with solution processed octadecyl substituted tetrabenzotriazaporphyrin as an active layer, IEEE Trans. Electron Devices 64 (6) (2017) 2629-2634.

[50] J. A. Jiménez-Tejada, P. Lopez-Varo, N. B. Chaure, I. Chambrier, A. N. Cammidge, M. J. Cook, A. Jafari-Fini, A. K. Ray, Organic thin film transistors using a liquid crystalline palladium phthalocyanine as active layer, J. Appl. Phys. 123 (11) (2018) 115501.

[51] K. M. Awawdeh, J. A. Jiménez-Tejada, P. López-Varo, J. A. LópezVillanueva, F. M. Gómez-Campos, M. J. Deen, Characterization of organic thin film transistors with hysteresis and contact effects, Org. Electron. 14 (12) (2013) 3286-3296.

[52] K. D. Holland, A. U. Alam, N. Paydavosi, M. Wong, C. M. Rogers, S. Rizwan, D. Kienle, M. Vaidyanathan, Impact of contact resistance on the $f_{t}$ and $f_{\max }$ of graphene versus $\mathrm{MoS}_{2}$ transistors, IEEE Trans. Nanotechnol. 16 (1) (2017) 94-106.

[53] S. V. Suryavanshi, E. Pop, S2DS: Physics-based compact model for circuit simulation of two-dimensional semiconductor devices including nonidealities, J. Appl. Phys. 120 (22) (2016) 224503.

[54] O. Marinov, M. J. Deen, C. Feng, Y. Wu, Precise parameter extraction technique for organic thin-film transistors operating in the linear regime, J. Appl. Phys. 115 (3) (2014) 034506.

[55] P. Lara Bullejos, J. A. Jiménez-Tejada, F. M. Gómez-Campos, M. J. Deen, O. Marinov, Evaluation of the charge density in the contact region of or- ganic thin film transistors, J. Appl. Phys. 106 (9) (2009) 094503.

[56] P. López-Varo, J. A. Jiménez-Tejada, J. A. López-Villanueva, J. E. Carceller, M. J. Deen, Modeling the transition from ohmic to space charge limited current in organic semiconductors, Org. Electron. 13 (9) (2012) 1700-1709.

[57] M. Higashiwaki, K. Konishi, K. Sasaki, K. Goto, K. Nomura, Q. T. Thieu, R. Togashi, H. Murakami, Y. Kumagai, B. Monemar, A. Koukitu, A. Kuramata, S. Yamakoshi, Temperature-dependent capacitance-voltage and current-voltage characteristics of pt/ga2o3 (001) schottky barrier diodes fabricated on $\mathrm{n}-\mathrm{ga} 2 \mathrm{o} 3$ drift layers grown by halide vapor phase epitaxy, Appl. Phys. Lett. 108 (13) (2016) 133503.

[58] M. Rapisarda, A. Valletta, A. Daami, S. Jacob, M. Benwadih, R. Coppard, G. Fortunato, L. Mariucci, Analysis of contact effects in fully printed pchannel organic thin film transistors, Org. Electron. 13 (10) (2012) 20172027.

[59] P. López-Varo, J. A. Jiménez-Tejada, J. A. López-Villanueva, M. J. Deen, Space-charge and injection limited current in organic diodes: A unified model, Org. Electron. 15 (10) (2014) 2526 - 2535.

[60] C. Rolin, E. Kang, J. H. Lee, G. Borghs, P. Heremans, J. Genoe, Charge carrier mobility in thin films of organic semiconductors by the gated van der Pauw method, Nat. Commun. 8 (2017) 14975.

[61] C.-H. Kim, S. Thomas, J. H. Kim, M. Elliott, J. E. Macdonald, M.-H. Yoon, Potentiometric parameterization of dinaphtho[2,3-b:2,,3/f]thieno[3,2-b]thiophene field-effect transistors with a varying degree of nonidealities, Advanced Electronic Materials 4 (7) (2018) 1700514

[62] J. R. Macdonald, Impedance spectroscopy, Annals of Biomedical Engineering 20 (3) (1992) 289-305.

[63] S. Luke, ECJ evolutionary computation library, available for free at http://cs.gmu.edu/ eclab/projects/ecj/ (1998).

[64] J. González, I. Rojas, J. Ortega, H. Pomares, F. Fernandez, A. Díaz, Multiobjective evolutionary optimization of the size, shape, and position parameters of radial basis function networks for function approximation, IEEE Trans. Neural Netw. 14 (6) (2003) 1478-1495.

[65] J. W. Borchert, B. Peng, F. Letzkus, J. N. Burghartz, P. K. L. Chan, K. Zojer, S. Ludwigs, H. Klauk, Small contact resistance and high-frequency operation of flexible low-voltage inverted coplanar organic transistors, Nat. Commun. 10 (1)

[66] V. Rajeev, A. K. Paulose, K. N. Unni, Ammonia gas detection using fieldeffect transistor based on a solution-processable organic semiconductor, Vacuum 158 (2018) 271-277. 